Pacific Journal of Mathematics

A REPRESENTATION THEOREM FOR ABELIAN GROUPS 


\title{
A REPRESENTATION THEOREM FOR ABELIAN GROUPS WITH NO ELEMENTS OF INFINITE P-HEIGHT
}

\author{
D. L. BOYER AND A. MADER
}

The purpose of this note is to give a generalization of the representation Theorems 33.1 and 33.2 of [2]. Let $G$ be an arbitrary abelian group and $B=\left[\bigoplus_{\lambda \in \Lambda}\left\langle x_{\lambda}\right\rangle\right] \oplus\left[\bigoplus_{i \geqq 1} B_{i}\right]$ be a $p$-basic subgroup of $G$, cf. [3], where $\bigoplus_{\lambda \in \Lambda}\left\langle x_{\lambda}\right\rangle$ is the torsionfree part. For all $\lambda \in A$ let $\left(F_{p}^{*}\right)_{\lambda}$ be a copy of the group of $p$-adic integers, and let $\left(F_{p}\right)_{\lambda}$ denote the infinite cyclic group of finite $p$-adic integers in $\left(F_{p}^{*}\right)_{\lambda}$. Then $G$ can be mapped homomorphically into the complete direct sum $\left[\bigoplus_{\lambda \in 1}^{*}\left(F_{p}^{*}\right)_{\lambda}\right] \oplus$ $\left[\bigoplus_{i \geqq 1}^{*} B_{i}\right]$ with kernel $p^{\omega} G$. Furthermore, the image of $G$ is a $p$-pure subgroup which contains $\left[\bigoplus_{\lambda \in \Lambda}\left(F_{p}\right)_{\lambda}\right] \oplus\left[\bigoplus_{i \geqq 1} B_{i}\right]$ as a $p$-basic subgroup and is in turn contained in the $p$-adic completion of this subgroup (See Section 1 for definitions). This representation is completely analogous to the representation theorem for $p$-groups which is contained as a special case, and hopefully it is of similar use.

Definitions and facts concerning p-adic and n-adic topologies. In this article we list the definitions and facts concerning $p$-adic and $n$-adic topologies that are needed in this paper. For references see $[2],[3]$, and [5].

Definition 1.1. The $p$-adic topology for an abelian group is the topology with the subgroups $p^{n} G, n=1,2, \cdots$ as a basis for the neighborhoods of 0 .

DeFInITION 1.2. The $n$-adic topology for an abelian group $G$ is the topology with the subgroups $n ! G, n=1,2, \cdots$ as a basis for the neighborhoods of 0 .

DEFINITION 1.3. The completion of an abelian group in the $p$-adic (resp. $n$-adic) topology is its metric space completion with respect to the metric $d(x, y)=10^{-m}$, where $m$ is the largest integer such that $x-y \varepsilon p^{m} G($ resp. $m ! G)$.

Proposition 1.4. If $H$ is a $p$-pure (resp. pure) subgroup of the abelian group $G$, then the $p$-adic (resp. $n$-adic) topology of the subgroup is the same as the induced $p$-adic (resp. n-adic) topology.

THEOREM 1.5. If an abelian group is complete in the n-adic topology, then it is a direct summand of every abelian group that 
contains it as a pure subgroup.

Proposition 1.6. A subgroup $H$ of an abelian group $G$ is dense in the $p$-adic (resp. $n$-adic) topology if and only if the quotient group $G / H$ is $p$-divisible (respectively divisible).

2. The representation theorems. Let $G$ be an abelian group, let $B$ be a $p$-basic subgroup, cf. Fuchs [3], of $G$, and we write $B=$ $\bigoplus_{n \geqq 0} B_{n}$ and $B_{0}=\bigoplus_{\lambda \in \Lambda}\left\langle x_{\lambda}\right\rangle$. As in [1 p. 325], for each $g \in G$ and each natural $n$, we can write

2.1. $g=b_{0}^{(n)}+b_{1}+\cdots+b_{n}+b_{n}^{*}+p^{n} g_{n}$ where $b_{0}^{(n)} \in B_{0}, \quad b_{i} \in B_{i}$ for $1 \leqq i \leqq n, b_{n}^{*} \in \bigoplus_{i>n} B_{i}$, and $g_{n} \in G$. It is proved in [1] p. 326 that the $b_{i}, i \geqq 1$, are unique in any such representation, and that, given two such representations, one for $n$ and one for $m$, we have

$$
b_{0}^{(n)}-b_{0}^{(m)} \in p^{\min (m, n)} G .
$$

For each $\lambda$, let $\left(F_{p}^{*}\right)_{\lambda}$ be the group of $p$-adic integers, and let $\left(F_{p}\right)_{\lambda}$ be the infinite cyclic subgroup of finite $p$-adic integers. We introduce the notation $P_{1}=\bigoplus_{\lambda \in A}^{*}\left(F_{p}^{*}\right)_{\lambda}$, and $P_{2}=\bigoplus_{i \geqq 1}^{*} B_{i} . \quad P_{1}$ and $P_{2}$ are complete groups in the $n$-adic topology, and the $n$-adic topology coincides with the $p$-adic topology. $\bigoplus_{\lambda \in_{A}}\left(F_{p}^{*}\right)_{\lambda}$ and $\bigoplus_{i \geq 1} B_{i}$ are pure subgroups of $P_{1}$ and $P_{2}$, hence they possess completions in $P_{1}$ and $P_{2}$ for the coinciding $n$-adic and $p$-adic topologies. Let $C_{1}=\left[\bigoplus_{\lambda \in \Lambda_{1}}\left(F_{p}^{*}\right)_{\lambda}\right]^{*}$ and $C_{2}=\left[\bigoplus_{i \geqq 1} B_{i}\right]^{*}$, where the *indicates the completion. Notice that $C_{i}$ is a direct summand of $P_{i}, i=1,2$.

We define a map $\sigma: G \rightarrow P_{1} \oplus P_{2}$ as follows. Let $g$ have the representation 2.1 for each $n$. Write $b_{0}^{(n)}=\sum_{\lambda \in \Lambda} m_{\lambda}^{(n)} x_{\lambda}$, and write $m_{\lambda}^{(n)}$ in its $p$-adic expansion

$$
m_{\lambda}^{(n)}=\sum_{k \geqq 0} a_{\lambda, k}^{(n)} p^{k}, 0 \leqq a_{\lambda, k}^{(n)} \leqq p-1 .
$$

It follows from 2.2 that $a_{\lambda, k}^{(n)}$ is independent of $n$ for $k<n$. Now define 2.4

$$
g \sigma=\left(\cdots, \sum_{k \geqq 0} a_{\lambda, k}^{(k+1)} p^{k}, \cdots ; b_{1}, b_{2}, \cdots\right) .
$$

Theorem 2.5. The map $\sigma$ is a homomorphism, and ker $\sigma=p^{\omega} G$, the subgroup of elements of infinite p-height. The p-basic subgroup $B$ of $G$ is mapped onto the group $\left[\bigoplus_{\lambda \in_{1}}\left(F_{p}\right)_{\lambda}\right] \oplus\left[\bigoplus_{i \geqq 1} B_{i}\right]$ which is a p-basic subgroup of $C_{1} \oplus C_{2}$.

Proof. It is easy to see that $\sigma$ is a homomorphism. Let $g \in p^{\omega} G$, and write $g$ as in 2.1. Then by the p-purity of $B$, each of $b_{0}^{(n)}$, $b_{1} \cdots, b_{n}, b_{n}^{*}$ is divisible by $p^{n}$ in the summand of $B$ to which it belongs. Hence $b_{1}=\cdots=b_{n}=0$. Since $b_{0}^{(n)}$ is divisible by $p^{n}$ in 
in $B_{0}$, it follows that in $m_{\lambda}^{(n)}=\sum_{k \geq 0} a_{\lambda, k}^{(n)} p^{k}$ the coefficient $\alpha_{\lambda, k}^{(n)}=0$ for $k \leqq n-1$. Thus $g \sigma=0$. Conversely, assume $g \sigma=0$. Then in the representation $2.1, b_{1}=b_{2}=\cdots=b_{n}=0$, and in the equation $m_{\lambda}^{(n)}=$ $=\sum_{k \gtrless \geq 0} \alpha_{\lambda, k}^{(n)} p^{k}, 0 \leqq a_{\lambda, k}^{(n)} \leqq p-1$, we have $a_{\lambda, k}^{(k+1)}=0$ for each $k$. The uniqueness of the $a_{\lambda, k}^{(n)}$ for $k<n$ implies $a_{\lambda, k}^{(n)}=0$ for $0 \leqq k<n$, i.e. $m_{\lambda}^{(n)}$ is divisible by $p^{n}$. Thus $b_{0}^{(n)}$ is divisible in $B_{0}$ by $p^{n}$. The remainder of this part of the proof is exactly as in the proof of Theorem 3 in [1] pp. 326-7. It is obvious from 2.1 that $B$ is mapped onto

$$
\left[\oplus_{\lambda \epsilon_{A}}\left(F_{p}\right)_{\lambda}\right] \oplus\left[\oplus_{i \geq 1} B_{i}\right],
$$

and it is easy to check that this is a p-basic subgroup of $C_{1} \oplus C_{2}$

Theorem 2.6. Go is p-pure in $P_{1} \oplus P_{2}$, and $(G \sigma)^{*}=C_{1} \oplus C_{2}$, where *indicates the completion in the p-adic topology.

Proof. By $2.5 B \sigma$ is a $p$-pure subgroup of $P_{1} \oplus P_{2}$. Since $G \sigma / B \sigma$ is a $p$-divisible (hence $p$-pure) subgroup of $\left(P_{1} \oplus P_{2}\right) / B \sigma$, it follows that $G \sigma$ is a $p$-pure subgroup of $P_{1} \oplus P_{2}$. Since $G \sigma$ is $p$-pure in $P_{1} \oplus P_{2}$ it possesses a $p$-adic completion in $P_{1} \oplus P_{2} . \quad B \sigma \leqq G \sigma$ implies $C_{1} \oplus C_{2}=$ $(B \sigma)^{*} \leqq(G \sigma)^{*}$, and since $B \sigma$ is dense in $G \sigma$ in the $p$-adic topology, $G \sigma \leqq(B \sigma)^{*}=C_{1} \oplus C_{2}$, thus $(G \sigma)^{*} \leqq C_{1} \oplus C_{2}$.

COROLlaRY 2.7. Every abelian group $G$ with no elements of infinite p-height may be considered to be a p-pure subgroup of some $\operatorname{group}\left[\bigoplus_{\lambda \in \Lambda}^{*}\left(F_{p}^{*}\right)_{\lambda}\right] \oplus\left[\bigoplus_{i \geqq 1}^{*} B_{i}\right]$ and containing $\left[\bigoplus_{\lambda \in \Lambda}\left(F_{p}\right)_{\lambda}\right] \oplus\left[\bigoplus_{i \geqq 1} B_{i}\right]$ as a p-basic subgroup.

If $G$ is a $p$-group, then $P_{1}=0$, and $G \sigma \leqq\left(C_{2}\right)_{t}$, the torsion subgroup of $C_{2}$. Thus in this case our theorems are exactly the important and useful Theorems 33.1 and 33.2 of [2].

\section{REFERENCES}

1. D. Boyer, On the theory of p-basic subgroups of abelian groups, Topics in abelian groups, Scott, Foresman and Company, 1963.

2. L. Fuchs, Abelian Groups, Pergamon Press, 1960.

3. Notes on abelian groups II, Acta Math. Acad. Sci. Hung. XI (1960), 117125.

4. D. Harrison, Infinite abelian groups and homological methods, Ann. of Math. (2) 69 (1959), 366-391.

5. I. Kaplansky, In finite abelian groups, University of Michigan Press, 1954.

Received May 14, 1965.

UNIVERSITY OF IDAHO 



\section{PACIFIC JOURNAL OF MATHEMATICS}

H. SAMELSON

Stanford University

Stanford, California

J. P. JaNs

University of Washington

Seattle, Washington 98105

\section{EDITORS}

J. DugundJI

University of Southern California Los Angeles, California 90007

RICHARD ARENS

University of California

Los Angeles, California 90024

\section{ASSOCIATE EDITORS}

E. F. BECKENBACH

B. H. NEUMANN

F. WOLF

K. YosidA

\section{SUPPORTING INSTITUTIONS}

UNIVERSITY OF BRITISH COLUMBIA

CALIFORNIA INSTITUTE OF TECHNOLOGY

UNIVERSITY OF CALIFORNIA

MONTANA STATE UNIVERSITY

UNIVERSITY OF NEVADA

NEW MEXICO STATE UNIVERSITY

OREGON STATE UNIVERSITY

UNIVERSITY OF OREGON

OSAKA UNIVERSITY

UNIVERSITY OF SOUTHERN CALIFORNIA
STANFORD UNIVERSITY

UNIVERSITY OF TOKYO

UNIVERSITY OF UTAH

WASHINGTON STATE UNIVERSITY

UNIVERSITY OF WASHINGTON

AMERICAN MATHEMATICAL SOCIETY CHEVRON RESEARCH CORPORATION TRW SYSTEMS

NAVAL ORDNANCE TEST STATION 


\section{Pacific Journal of Mathematics}

\section{Vol. 20, No. $1 \quad$ September, 1967}

Leonard Daniel Baumert, Extreme copositive quadratic forms. II ........ 1

Edward Lee Bethel, A note on continuous collections of disjoint

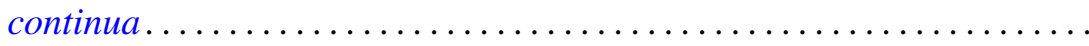

Delmar L. Boyer and Adolf G. Mader, A representation theorem for abelian groups with no elements of infinite p-height ...................

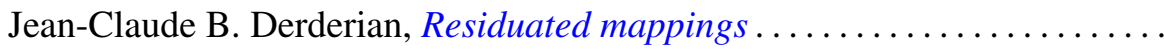

Burton I. Fein, Representations of direct products of finite groups ......... 45

John Brady Garnett, A topological characterization of Gleason parts.......

Herbert Meyer Kamowitz, On operators whose spectrum lies on a circle or

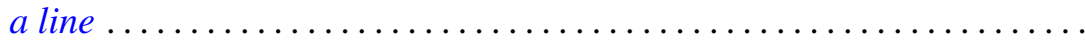

Ignacy I. Kotlarski, On characterizing the gamma and the normal distribution ........................................

Yu-Lee Lee, Topologies with the same class of homeomorphisms ..........

Moshe Mangad, Asymptotic expansions of Fourier transforms and discrete polyharmonic Green's functions ...........................

Jürg Thomas Marti, On integro-differential equations in Banach spaces ....

Walter Philipp, Some metrical theorems in number theory.............. 109

Maxwell Alexander Rosenlicht, Another proof of a theorem on rational

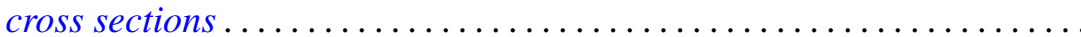

Kenneth Allen Ross and Karl Robert Stromberg, Jessen's theorem on Riemann sums for locally compact groups

Stephen Simons, A theorem on lattice ordered groups, results of Ptak, Namioka and Banach, and a front-ended proof of Lebesgue's theorem...

Morton Lincoln Slater, On the equation $\varphi(x)=\int_{x} x+1 K(\xi) f[\varphi(\xi)] d \xi \ldots$ 155 Arthur William John Stoddart, Existence of optimal controls .. 167 Burnett Roland Toskey, A system of canonical forms for rings on a direct sum of two infinite cyclic groups ....................

Jerry Eugene Vaughan, A modification of Morita's characterization of dimension 